\title{
Postradiation cutaneous angiosarcoma after treatment of breast carcinoma is characterized by MYC amplification in contrast to atypical vascular lesions after radiotherapy and control cases: clinicopathological, immunohistochemical and molecular analysis of 66 cases
}

\author{
T Mentzel ${ }^{1}$, HU Schildhaus ${ }^{2}$, G Palmedo ${ }^{1}$, R Büttner ${ }^{2}$ and H Kutzner ${ }^{1}$ \\ ${ }^{1}$ Dermatopathologie Bodensee, Friedrichshafen, Germany and ${ }^{2}$ Institute of Pathology, University Hospital \\ Cologne, Cologne, Germany
}

\begin{abstract}
Postradiation cutaneous vascular lesions after treatment of breast carcinoma comprise a heterogeneous group of benign, atypical, and malignant lesions and are best regarded as points along a morphological spectrum. We analyzed a series of cutaneous angiosarcomas after treatment of breast cancer in comparison with control cases and cases of atypical vascular lesions with special emphasis on the expression and amplification of MYC. The 66 cases were divided into control cases (5), cases in which a slight vascular proliferation was seen after radiotherapy of breast cancer (12), cases of atypical vascular lesions after radiotherapy (16), cases of postradiation cutaneous angiosarcomas (25), and cases of angiosarcomas of skin and soft tissues unrelated to radiotherapy (8). None of the control cases (2 M, 3 F, 20-76 years), of cases showing slight vascular proliferation, dermal fibrosis and inflammation after radiotherapy of breast cancer (12 F, 48-79 years), of cases of atypical vascular lesions after radiotherapy (16 F, 29-81 years), and of cases of angiosarcomas of skin and soft tissues unrelated to radiotherapy (3 M, 5 F, 25-92 years) showed an amplification of $M Y C$ by FISH analysis. In striking contrast, in all cases of postradiation cutaneous angiosarcomas (25 F, 46-95 years), MYC amplification was found by FISH analysis in a variable number of counted nuclei. Immunohistochemically, strong positive nuclear staining for MYC and prox-1 was seen in cases of postradiation cutaneous angiosarcoma, whereas control cases and cases of atypical vascular proliferation after radiotherapy were negative for MYC, and stained only focally positive for prox-1 in a number of cases. In conclusion, the presence of $M Y C$ amplification represents an important additional diagnostic tool in the distinction of postradiation cutaneous angiosarcomas from atypical vascular lesions after radiotherapy. Immunohistochemical stainings for $M Y C$ are useful for mapping of these lesions and for careful tumor margin control.

Modern Pathology (2012) 25, 75-85; doi:10.1038/modpathol.2011.134; published online 9 September 2011
\end{abstract}

Keywords: angiosarcoma; atypical vascular lesion; breast carcinoma; FISH; MYC

Correspondence: Professor Dr T Mentzel, MD, Dermatopathologie, Friedrichshafen, Siemensstrasse 6/1, Friedrichshafen D-88048, Germany.

E-mail: mentzel@dermpath.de or www.dermpath.de

Received 13 April 2011; revised 7 June 2011; accepted 7 June 2011; published online 9 September 2011
Cutaneous angiosarcomas occur in three main clinical settings: so-called idiopathic angiosarcoma arising most frequently in sun-damaged skin of the face in elderly patients, rare lymphedematous angiosarcoma arising in patients with chronic lymphedema, and postradiation cutaneous angiosarcoma, usually occurring in patients with a history 
of radiation therapy for breast cancer. In addition to postradiation angiosarcoma, benign and atypical vascular proliferations after radiotherapy have been described. Benign lymphangiomatous papule usually presents as a solitary, well-circumscribed, and superficially located dermal neoplasm that is composed of dilated, lymphangioma-like vascular spaces lined by slightly enlarged but uniform endothelial cells without increased atypia and proliferative activity, and endothelial multilayering is usually not present. ${ }^{1,2}$ Atypical vascular lesions following radiotherapy for breast cancer are solitary or multicentric vascular lesions composed of either superfially located, lymphangioma-like vascular lesions or of narrow and dissecting vascular proliferations that may show focally anastomosing spaces. The lining endothelial tumor cells may contain hyperchromatic nuclei, but significant cytologic atypia, an increased mitotic rate and infiltration of subcutaneous tissue are not seen. ${ }^{3}$ During the last 15 years it has been shown that there is significant clinical and morphological overlap between benign, atypical, and malignant vascular proliferations after radiotherapy, neoplasms that are best regarded as points along a morphological spectrum, and that exact classification especially on a small biopsy may cause significant diagnostic problems. ${ }^{4}$ In addition, shared mutations of the p53 tumor suppressor gene emphasize that atypical and malignant radiation-induced vascular changes are closely related. ${ }^{5}$ Most recently it has been reported, that so-called secondary cutaneous angiosarcomas (postradiation angiosarcomas and lymphedemaassociated angiosarcomas) show high-level amplifications of $M Y C$ at locus 8q24.21 in contrast to primary cutaneous angiosarcomas. ${ }^{6}$ We decided to study benign, atypical, and malignant vascular proliferations after radiotherapy in order to compare immunohistochemical expression of MYC and the presence or absence of $M Y C$ amplifications in these lesions.

\section{Materials and methods}

The cases were retrieved from the routine files of the Department of Pathology, University of Bonn, the Dermatopathologie Bodensee, Friedrichshafen, and from referral files of three of the authors (TM, HUS, and $\mathrm{HK}$ ), and clinical and follow-up information were obtained from the referring pathologists. As control cases classical hemangiomas and cases of postradiation changes without significant vascular proliferation were chosen. In addition, some cases of spontaneous angiosarcoma were added for comparison. The tissue was fixed in $4 \%$ buffered formalin in all cases, routinely processed and embedded in paraffin and 2-4 $\mu \mathrm{m}$ thick sections were stained with hematoxylin and eosin. In addition, representative sections were stained immunohistochemically by the labeled streptavidin biotin technique using commercially available antibodies; antigen retrieval was used for all antibodies. Stainings for CD 31 (clone JC70A, dilution 1:100, DAKO, Hamburg, Germany), $\alpha$-smooth muscle actin (1A4, 1:500, DAKO), Ki-67 (SP6, 1:300, Roche Diagnostics, Mannheim, Germany), MYC (Y69, 1:100, Biomol, Hamburg, Germany), and prox-1 (polyclonal, 1:200, RELIATech, Wolfenbüttel, Germany) were available in most cases. Appropriate positive and negative controls were used. Fluorescence in situ hybridization was performed on $3 \mu \mathrm{M}$ sections of formalin-fixed, paraffin-embedded tissue after deparaffinization with xylene, dehydration by graded ethanol and air-drying. All tissue sections were pretreated and digested as recommended by the probe supplier. Digestion times were optimized on a case-by-case basis. After pretreatment and digestion steps, sections were washed in $2 \times$ SSC, $\mathrm{pH} 7.0$, for $2 \mathrm{~min}$ at $45^{\circ} \mathrm{C}$. The MYC probe (Vysis LSI C-MYC, Abbott, Wiesbaden, Germany) was applied to the sections and the coverslides were sealed with rubber cement, heat-denatured for $10 \mathrm{~min}$ and hybridized at $37^{\circ} \mathrm{C}$ for $16 \mathrm{~h}$ or overnight. Cohybridization with the centromeric enumeration probe (CEP8, D8Z2, Abbott) was carried out in a subset of cases. Stringent washing was performed as recommended by the probe supplier. All sections were counterstained with DAPI II in mounting medium $(125 \mathrm{ng} / \mathrm{ml}$, Abbott). A minimum of 45 non-overlapping intact nuclei were assessed for the presence of amplifications; an amplification was defined as multiple clustered signals ( $>8$ signals) according to the previously described findings. ${ }^{6}$ FISH slides were evaluated by two experienced investigators (GP, HUS) using appropriate microscopes and filter sets. After initial investigation, all specimens were cross-validated by GP and HUS in a consensus meeting. For photographic documentation, nuclei with the appropriate signals were digitally photographed with a monochrome RT3 CCD camera (Diagnostic Instruments, Sterling Heights, USA) connected to a Zeiss Axioplan 2 microscope (Zeiss, Oberkochen, Germany) using a HBO103 lamp and the appropriate filters (spectrum orange and 4,6-diamino-2-phenylindole) for the two fluorescence dyes (Zeiss). Reconstruction into a single superimposed image with blue and orange pseudocolors was accomplished using the SPOT software (Visitron Systems, Puchheim, Germany).

\section{Results}

The clinical details of the affected patients are summarized in Table 1, and selected results of immunohistochemical investigations and of FISH analysis are listed in Table 2.

\section{Control Group (Cases 1-5)}

Five patients $(2 \mathrm{M}, 3 \mathrm{~F}$, age ranged between 20 and 76 years) were included into the control group. 
Table 1 Clinical findings of 66 patients with radiation-induced vascular lesions

\begin{tabular}{|c|c|c|c|c|c|}
\hline Case & $G d$ & Age (ys.) & Location & Diagnosis & Treatment/follow-up \\
\hline 1 & $\mathrm{~F}$ & 36 & Buttock & $\mathrm{HE}$ & Excision \\
\hline 2 & M & 20 & Leg & Hobnail HE & Excision \\
\hline 3 & $\mathrm{~F}$ & 76 & Rectum & Proctitis & Biopsy \\
\hline 4 & $\mathrm{M}$ & 71 & Rectum & Proctitis & Biopsy \\
\hline 5 & $\mathrm{~F}$ & 70 & Breast & BCC & Excision \\
\hline 6 & $\mathrm{~F}$ & 54 & Breast & Increase of vessels & Biopsy \\
\hline 7 & $\mathrm{~F}$ & 49 & Breast & Increase of vessels & Biopsy \\
\hline 8 & $\mathrm{~F}$ & 67 & Breast & Ulceration, radiodermatitis & Excision \\
\hline 9 & $\mathrm{~F}$ & 49 & Breast & Radiodermatitis, erosion & Excision \\
\hline 10 & $\mathrm{~F}$ & 74 & Breast & Radiodermatitis & Biopsy \\
\hline 11 & $\mathrm{~F}$ & 71 & Breast & Radiodermatitis & Biopsy \\
\hline 12 & $\mathrm{~F}$ & 65 & Breast & Radiodermatitis & Biopsy \\
\hline 13 & $\mathrm{~F}$ & 76 & Breast & Ulceration, radiodermatitis & Excision \\
\hline 14 & $\mathrm{~F}$ & 74 & Breast & Radiodermatitis & Biopsy \\
\hline 15 & $\mathrm{~F}$ & 48 & Breast & Radiodermatitis & Biopsy \\
\hline 16 & $\mathrm{~F}$ & 51 & Breast & Radiodermatitis & Biopsy \\
\hline 17 & $\mathrm{~F}$ & 79 & Breast & Fibrosis, few vessels & Biopsy \\
\hline 18 & $\mathrm{~F}$ & 44 & Breast & AVL & CE, NSR at $15 / 12$ \\
\hline 19 & $\mathrm{~F}$ & 63 & Axilla & BLAP & CE, NSR at $12 / 12$ \\
\hline 20 & $\mathrm{~F}$ & 55 & Breast & AVL & CE, no follow-up \\
\hline 21 & $\mathrm{~F}$ & 55 & Breast & AVL (2 lesions) & CE, no follow-up \\
\hline 22 & $\mathrm{~F}$ & 65 & Breast & AVL & CE, NSR at $84 / 12$ \\
\hline 23 & $\mathrm{~F}$ & 60 & Breast & AVL & CE, NSR at $32 / 12$ \\
\hline 24 & $\mathrm{~F}$ & 81 & Breast & AVL & Biopsy, no follow-up \\
\hline 25 & $\mathrm{~F}$ & 65 & Breast & AVL & $\begin{array}{l}\text { CE, R at } 12 \text { months, CE } \\
\text { NSR } 60 \text { months later }\end{array}$ \\
\hline 26 & $\mathrm{~F}$ & 64 & Breast & BLAP & CE, no follow-up \\
\hline 27 & $\mathrm{~F}$ & 58 & Breast & AVL & $\begin{array}{l}\text { Biopsy, multiple lesions, appear and } \\
\text { disappear, no progression at } 36 / 12\end{array}$ \\
\hline 28 & $\mathrm{~F}$ & 57 & Breast & AVL & CE, NSR at $144 / 12$ \\
\hline 29 & $\mathrm{~F}$ & 29 & Breast & AVL & CE, NSR at $27 / 12$ \\
\hline 30 & $\mathrm{~F}$ & 65 & Breast & AVL & CE, NSR at $24 / 12$ \\
\hline 31 & $\mathrm{~F}$ & 46 & Breast & AVL (multiple lesions) & Multiple biopsies, no progression at $12 / 12$ \\
\hline 32 & $\mathrm{~F}$ & 40 & Breast & AVL & CE, no follow-up \\
\hline 33 & $\mathrm{~F}$ & 51 & Breast & AVL (two lesions) & CE, NSR at $18 / 12$ \\
\hline 34 & $\mathrm{~F}$ & 72 & Upper arm & Angiosarcoma of ST & CE, no follow-up \\
\hline 35 & $\mathrm{M}$ & 92 & Lower leg & Cutaneous angiosarcoma & CE, no follow-up \\
\hline 36 & M & 25 & NA & Angiosarcoma of ST & CE, no follow-up \\
\hline 37 & $\mathrm{M}$ & 61 & Neck & Angiosarcoma of ST & Marginal excision, no follow-up \\
\hline 38 & $\mathrm{~F}$ & 51 & Chest wall & Angiosarcoma of ST & $\mathrm{R}$ at $18 / 12$ \\
\hline 39 & $\mathrm{~F}$ & 46 & Breast & Angiosarcoma of ST & CE, MTS at $110 / 12$ \\
\hline 40 & $\mathrm{~F}$ & 43 & Breast & Angiosarcoma of ST & CE, no follow-up \\
\hline 41 & $\mathrm{~F}$ & 50 & NA & Angiosarcoma of ST & CE, no follow-up \\
\hline 42 & $\mathrm{~F}$ & 65 & Breast & Cutaneous angiosarcoma & $\mathrm{CE}, \mathrm{R}$ at $7 / 12$, CT \\
\hline 43 & $\mathrm{~F}$ & 67 & Breast & Cutaneous angiosarcoma & CE, NSR at $10 / 12$ \\
\hline 44 & $\mathrm{~F}$ & 73 & Breast & Cutaneous angiosarcoma & CE, $R$ at $30 / 12, D O D$ at $34 / 12$ \\
\hline 45 & $\mathrm{~F}$ & 84 & Breast & Cutaneous angiosarcoma & CE, NSR at $31 / 12$ \\
\hline 46 & $\mathrm{~F}$ & 95 & Breast & Cutaneous angiosarcoma & Biopsy, lost to follow-up \\
\hline 47 & $\mathrm{~F}$ & 75 & Breast & Cutaneous angiosarcoma & CE, NSR at $12 / 12$ \\
\hline 48 & $\mathrm{~F}$ & 64 & Breast & Cutaneous angiosarcoma & CE, no follow-up \\
\hline 49 & $\mathrm{~F}$ & 63 & Breast & Cutaneous angiosarcoma & $\mathrm{CE}, \mathrm{R}$ at $36 / 12$, NSR at $12 / 12$ \\
\hline 50 & $\mathrm{~F}$ & 75 & Breast & Cutaneous angiosarcoma & CE, $R$ at $18 / 12, N S R$ at $5 / 12$ \\
\hline 51 & $\mathrm{~F}$ & 56 & Breast & Cutaneous angiosarcoma & CE, R, MTS, DOD at 60/12 \\
\hline 52 & $\mathrm{~F}$ & 83 & Breast & Cutaneous angiosarcoma & DOD at $5 / 12$ \\
\hline 53 & $\mathrm{~F}$ & 63 & Breast & Cutaneous angiosarcoma & CE, MTS, DOD at $8 / 12$ \\
\hline 54 & $\mathrm{~F}$ & 70 & Breast & Cutaneous angiosarcoma & CE, CT, DOD at $24 / 12$ \\
\hline 55 & $\mathrm{~F}$ & 62 & Breast & Cutaneous angiosarcoma & CE, no follow-up \\
\hline 56 & $\mathrm{~F}$ & 46 & Breast & Cutaneous angiosarcoma & Biopsy, no follow-up \\
\hline 57 & $\mathrm{~F}$ & 68 & Breast & Cutaneous angiosarcoma & $\mathrm{CE}, \mathrm{R}$ and lymph node MTS, DOD at $24 / 12$ \\
\hline 58 & $\mathrm{~F}$ & 70 & Breast & Cutaneous angiosarcoma & CE, NSR at $30 / 12$ \\
\hline 59 & $\mathrm{~F}$ & 74 & Breast & Cutaneous angiosarcoma & CE, NSR at 7/12 \\
\hline 60 & $\mathrm{~F}$ & 68 & Breast & Cutaneous angiosarcoma & CE, NSR at $8 / 12$ \\
\hline 61 & $\mathrm{~F}$ & 78 & Breast & Cutaneous angiosarcoma & $\mathrm{CE}, \mathrm{R}$ at $9 / 12$ \\
\hline 62 & $\mathrm{~F}$ & 66 & Breast & Cutaneous angiosarcoma & CE, no follow-up \\
\hline 63 & $\mathrm{~F}$ & 57 & Breast & Cutaneous angiosarcoma & CE, NSR at $28 / 12$ \\
\hline 64 & $\mathrm{~F}$ & 69 & Breast & Cutaneous angiosarcoma & CE, NSR at 5/12 \\
\hline 65 & $\mathrm{~F}$ & 71 & Breast & Cutaneous angiosarcoma & CE, recent case \\
\hline 66 & $\mathrm{~F}$ & 54 & Breast & Cutaneous angiosarcoma & $\mathrm{CE}$, recent case \\
\hline
\end{tabular}

Gd, gender; F, female; M, male; HE, hemangioma; BCC, basal cell carcinoma; AVL, atypical vascular lesion; BLAP, benign lymphangiomatous papule; CE, complete excision; R, local recurrence; MTS, metastases; DOD, died of disease; NSR, no sign of recurrence; ST, soft tissue; NA, not available; CT, polychemotherapy. 
Table 2 Immunohistochemical findings and FISH analysis of 66 patients with radiation-induced vascular lesions

\begin{tabular}{|c|c|c|c|}
\hline Case & $I M M Y C$ & IM prox-1 & FISH MYC \\
\hline 1 & - & - & NAMP \\
\hline 2 & - & Focal + & NAMP \\
\hline 3 & - & - & NAMP \\
\hline 4 & - & - & NAMP \\
\hline 5 & NA & NA & NAMP \\
\hline 6 & - & - & NAMP \\
\hline 7 & - & Focal + & \\
\hline 8 & - & - & NAMP \\
\hline 9 & - & - & NAMP \\
\hline 10 & - & - & NAMP \\
\hline 11 & - & - & NAMP \\
\hline 12 & - & - & NAMP \\
\hline 13 & - & - & NAMP \\
\hline 14 & - & - & NAMP \\
\hline 15 & NA & NA & NAMP \\
\hline 16 & - & - & NAMP \\
\hline 17 & - & - & NAMP \\
\hline 18 & - & + & NAMP \\
\hline 19 & - & Focal + & \\
\hline 20 & - & Focal + & \\
\hline 21 & - & - & NAMP \\
\hline 22 & - & Focal + & \\
\hline 23 & - & - & NAMP \\
\hline 24 & - & Focal + & NAMP \\
\hline 25 & - & Focal + & NAMP \\
\hline 26 & - & Focal + & NAMP \\
\hline 27 & - & Focal + & NAMP \\
\hline 28 & Focal + & & NAMP \\
\hline 29 & - & - & NAMP \\
\hline 30 & - & Focal + & NAMP \\
\hline 31 & - & - & NAMP \\
\hline 32 & - & Focal + & NAMP \\
\hline 33 & - & Focal + & NAMP \\
\hline 34 & - & - & NAMP \\
\hline 35 & - & - & NAMP \\
\hline 36 & - & - & NAMP \\
\hline 37 & - & - & NAMP \\
\hline 38 & - & - & NAMP \\
\hline 39 & - & - & NAMP \\
\hline 40 & - & - & NAMP \\
\hline 41 & - & Focal + & NAMP \\
\hline 42 & + & + & AMP in $46 / 52$ \\
\hline 43 & + & Focal + & AMP in $12 / 54$ \\
\hline 44 & + & - & AMP in $11 / 51$ \\
\hline 45 & + & + & AMP in $48 / 54$ \\
\hline 46 & + & - & AMP in $43 / 51$ \\
\hline 47 & + & + & AMP in $39 / 50$ \\
\hline 48 & + & + & AMP in $12 / 56$ \\
\hline 49 & + & + & AMP in $43 / 55$ \\
\hline 50 & + & + & AMP in $46 / 52$ \\
\hline 51 & + & + & AMP in $18 / 50$ \\
\hline 52 & + & + & AMP in $29 / 50$ \\
\hline 53 & + & + & AMP in $48 / 55$ \\
\hline 54 & + & + & AMP in $50 / 58$ \\
\hline 55 & - & Focal + & AMP in $9 / 45$ \\
\hline 56 & NA & NA & AMP in $7 / 50$ \\
\hline 57 & + & + & AMP in $37 / 51$ \\
\hline 58 & Focal + & - & AMP in $8 / 49$ \\
\hline 59 & NA & NA & AMP in $15 / 49$ \\
\hline 60 & + & + & AMP in $21 / 50$ \\
\hline 61 & + & - & AMP in $79 / 100$ \\
\hline 62 & + & - & AMP in $84 / 100$ \\
\hline 63 & + & - & AMP in $85 / 100$ \\
\hline 64 & + & NA & AMP in $24 / 49$ \\
\hline 65 & + & NA & AMP in $48 / 50$ \\
\hline 66 & NA & NA & AMP in $50 / 50$ \\
\hline
\end{tabular}

IM, immunohistochemistry; NA, data not available; NAMP, no amplification; AMP, MYC amplification in out of the nuclei counted.
In Case 1, a hemangioma arising on the buttock was completely excised. The patient in Case 2 developed a well-circumscribed hobnail hemangioma in the popliteal fossa, showing a biphasic growth of superficially dilated, lymphangioma-like vascular structures, and deeper rather narrow vessels lined by hobnail endothelial cells containing enlarged but uniform nuclei. Cases 3 and 4 contained biopsies from the rectum showing moderate postradiation colitis after radiotherapy of rectal and prostatic cancers. The patient in Case 5 is a 70-year-old female who developed a basal cell carcinoma in the postradiation area, who previously received radiotherapy for breast cancer with dermal fibrosis, slight vascular proliferation, and scattered inflammatory cells. Immunohistochemically, the endothelial cells of the hobnail hemangioma in Case 2 stained focally positive for prox-1, whereas the remaining cases were negative, and endothelial cells did not stain positively for MYC. In none of the control cases, an amplification of $M Y C$ was detected by FISH analysis.

\section{Cases Showing Radiation-Induced Vascular Proliferation (Cases 6-17)}

This group comprised 12 female patients (age ranged between 48 and 79 years), who received radiotherapy for breast cancer. All carcinomas were excised completely, and the vascular proliferation developed within 1 and 12 years after radiotherapy. The patients presented clinically with ill-defined, sometimes slightly indurated and erythematous skin areas in the postradiation areas, and skin ulceration was seen in two cases (Cases 8 and 13). Histologically, a slight and diffuse increase of dilated or narrow vessels associated with dermal fibrosis and scattered inflammatory cells was seen in all cases, and in Cases 9-16 the dermal fibrosis was more intense. Immunohistochemically, all cases were negative for MYC, and focal expression of prox-1 was noted in only one case (Case 7). No $M Y C$ amplification was detected by FISH analysis.

\section{Atypical Vascular Proliferations after Radiotherapy (Cases 18-33)}

In this group 16 female patients were included (age ranged between 29 and 81 years), who received radiotherapy for breast cancer and developed an atypical vascular proliferation within 2 and 8 years after radiotherapy. In Case 21 two lesions were seen, and in Cases 27 and 31 multiple lesions developed within a short time period (Figure 1), the remaining patients presented with solitary lesions. The lesions were excised completely and no progression to an angiosarcoma was reported. Follow-up information was available in 11 patients (range from 12 to 144 months) and revealed a local recurrence in Case 25 who was treated with complete excision. 


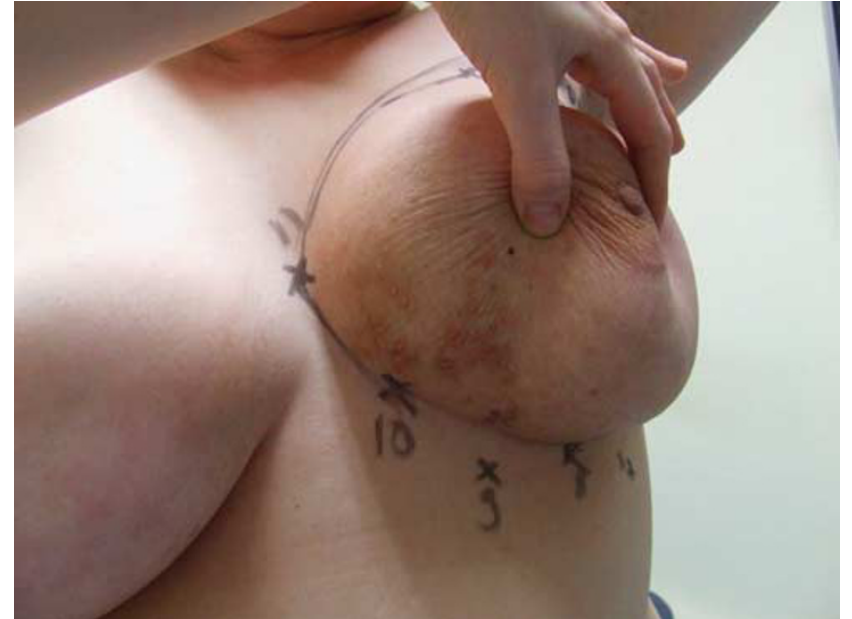

Figure 1 The patient in Case 31 presented with multiple lesions. Numerous lesions were excised, and no progression within 12 months was noted.
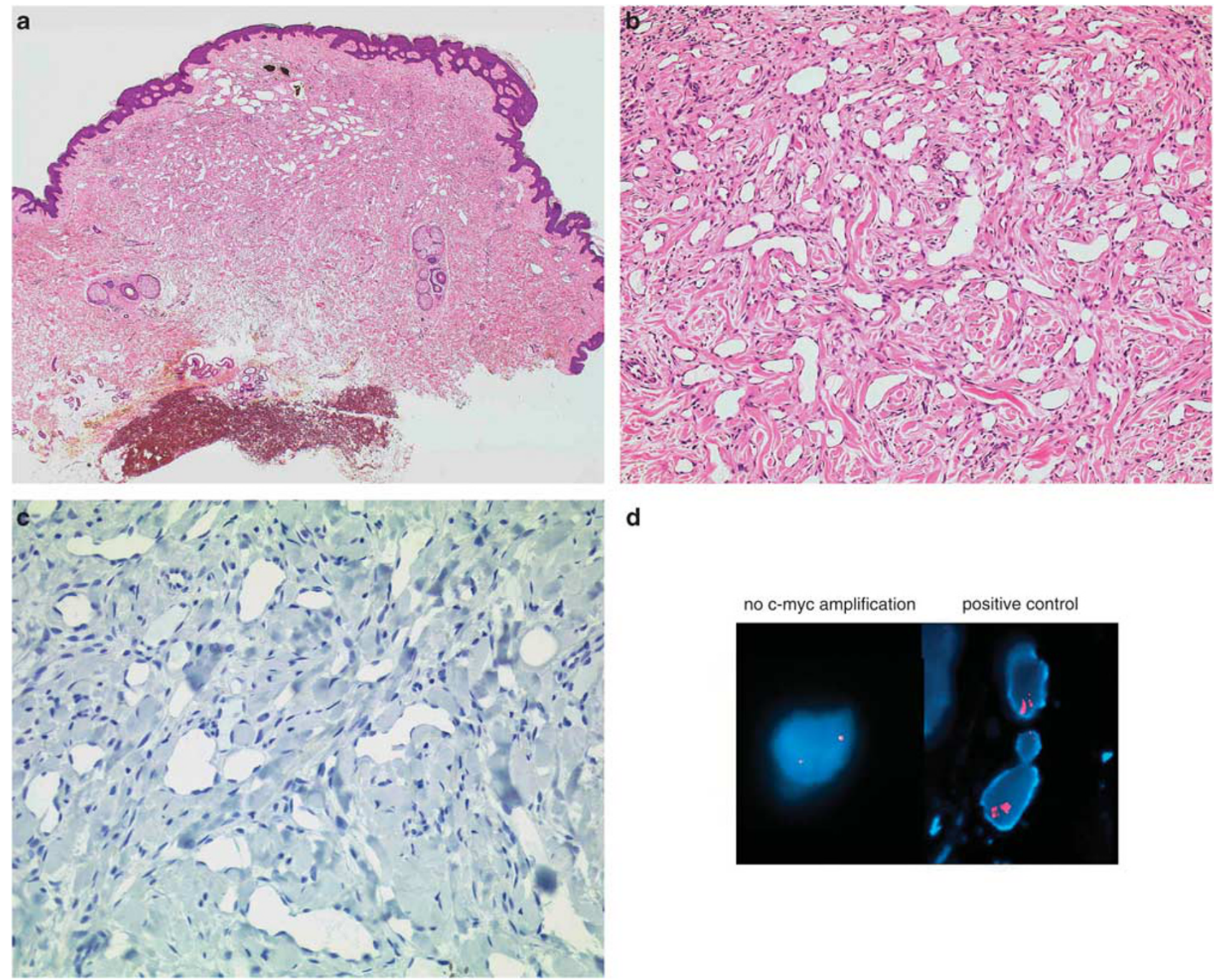

d

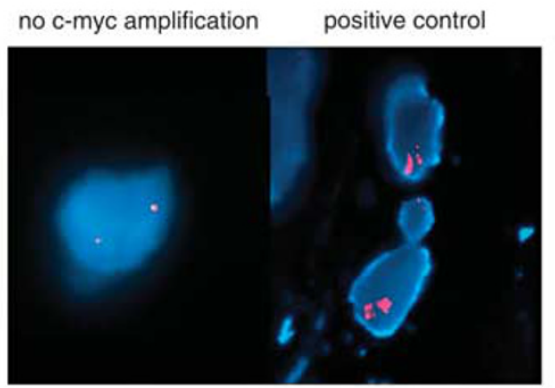

Figure 2 Case 19 showed morphological features of benign lymphangiomatous papule with dilated, lymphangioma-like vascular structures (a). Higher power view reveals dilated vascular structures lined by bland endothelial cells (b). Immunohistochemically, no expression of MYC was seen in endothelial tumor cells (c). No MYC amplification was detected by FISH analysis (d). 
vascular structures with stromal fibrosis. The remaining cases were composed of rather illdefined, superficially located dermal vascular lesions composed of dilated and narrow vascular structures. In Case 31, multiple lesions were excised showing features of either well-circumscribed lymphangioma or of ill-defined vascular lesions composed of narrow vascular structures. The lining endothelial cells contained slightly enlarged but relatively uniform, sometimes hyperchromatic, nuclei, and no increased proliferative activity was detected. In Cases 18, 22, 23, 25, 27, 30, and 33 more worrisome morphological features were present. At least focally, anastomosing vascular spaces were noted, and focal endothelial multilayering was present in these lesions (Figure 3). Immunohistochemically, only few endothelial cells showed Ki-67 expression. Whereas a focal expression of prox-1 was noted in 10 cases (Cases 18, 20, 22, 24, 25, 26, $27,30,32$, and 33), only few endothelial cells in
Case 28 stained positively for MYC, the remaining cases were negative. No $M Y C$ amplification was detected in any case (Figure 3).

\section{Angiosarcomas of Skin and Soft Tissues Unrelated to Radiotherapy (Cases 34-41)}

Three male and five female patients (age ranged between 25 and 92 years) in this group developed an angiosarcoma unrelated to previous radiotherapy. The neoplasms of Cases 34, 36, 37, 38, and 41 arose in subcutaneous and deep soft tissues of the trunk, the neck and the extremities, Case 35 represented a cutaneous angiosarcoma arising on the lower leg, and Cases 39 and 40 arose in deep tissue of the breast. Histologically, the neoplasms showed a broad morphological spectrum ranging from welldifferentiated angiosarcoma in Case 40 to poorly differentiated angiosarcoma composed of sheets of
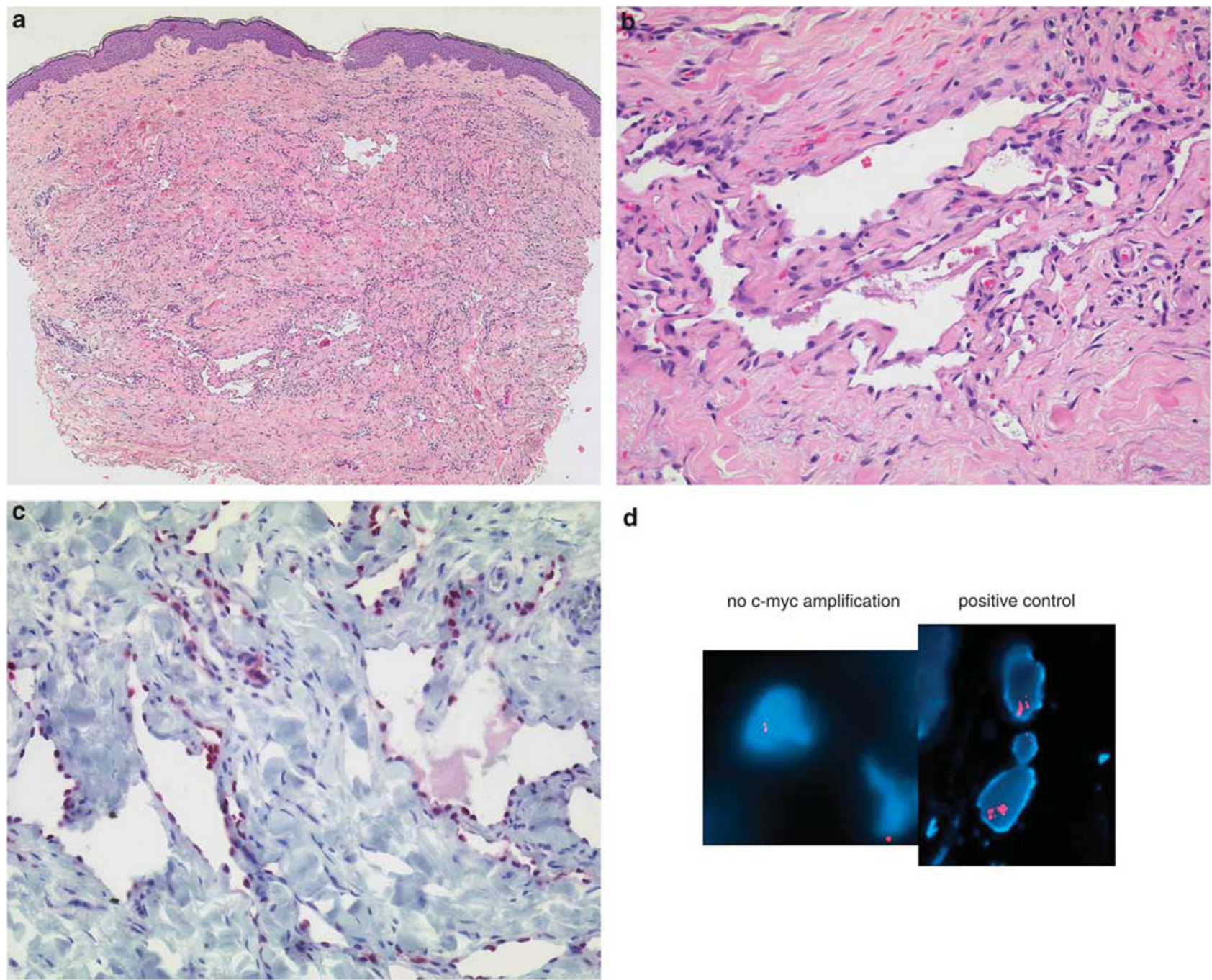

d

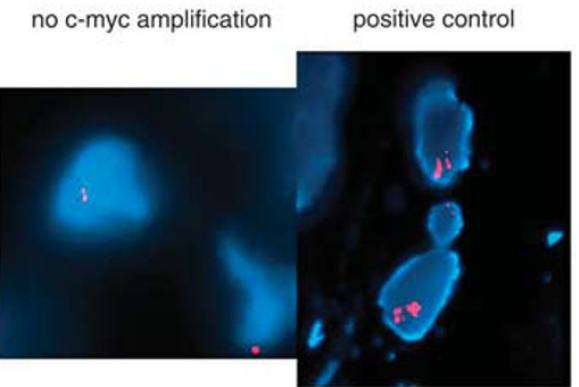

Figure 3 An atypical vascular lesion was seen in Case 18. The rather ill-defined dermal neoplasm is composed of narrow and dilated vascular structures dissecting the dermis (a). The vascular spaced are lined by endothelial cells with enlarged but uniform nuclei. No endothelial multilayering and no significant atypia are present (b). A focal expression of prox-1 by endothelial cells was noted (c). No $M Y C$ amplification was detected by FISH analysis (d). 
atypical endothelial tumor cells with numerous mitoses and areas of tumor necrosis in Case 34. A predominantly spindle cell angiosarcoma was seen in Case 36. Unfortunately, follow-up information was available in only two patients. A local recurrence at 18 months occurred in Case 38, and the patient of Case 39 developed multiple liver and peritoneal metastases at 110 months. Immunohistochemically, focal expression of prox-1 was noted in Case 41. No $M Y C$ amplification was detected in any case.

\section{Angiosarcomas after Radiotherapy (Cases 42-66)}

In this group 25 female patients, who developed a cutaneous angiosarcoma after radiotherapy for breast cancer, were included. In all cases the breast carcinoma had been excised completely, and the vascular neoplasms developed within 1.5 and 13 years after radiotherapy (Figure 4). The age of the affected patient ranged from 46 to 95 years. Followup information of 19 patients (range from 5 to 60 months) revealed local recurrences in seven cases (Cases 42, 44, 49, 50, 51, 57, and 61), lymph node metastases in Case 57, multiple metastases in two patients (Cases 51 and 53), and six patients died of disease (Cases 44, 51, 52, 53, 54, and 57).

In 21 patients a dermal angiosarcoma was seen, whereas in Cases 48, 52, 58, and 62 the dermis and the subcutis were involved. Histologically, a broad morphological spectrum was present ranging from well-differentiated, BLAP- or AVL-like cutaneous angiosarcomas in Cases 55 and 59 to poorly differentiated neoplasms, i.e., in Case 62. The neoplasms were composed of dissecting, infiltrating, and anastomosing vascular structures lined by enlarged endothelial tumor cells containing enlarged vesicular or hyperchromatic nuclei (Figure 5). A solid growth was present in Cases 46, 52, 62, and 65 . Whereas mild cytologic atypia was present in Case 55, severe atypia was noted in Cases 45, 52, 62, 64, and 65. Interestingly, numerous vascular tufts resembling morphological features of tufted

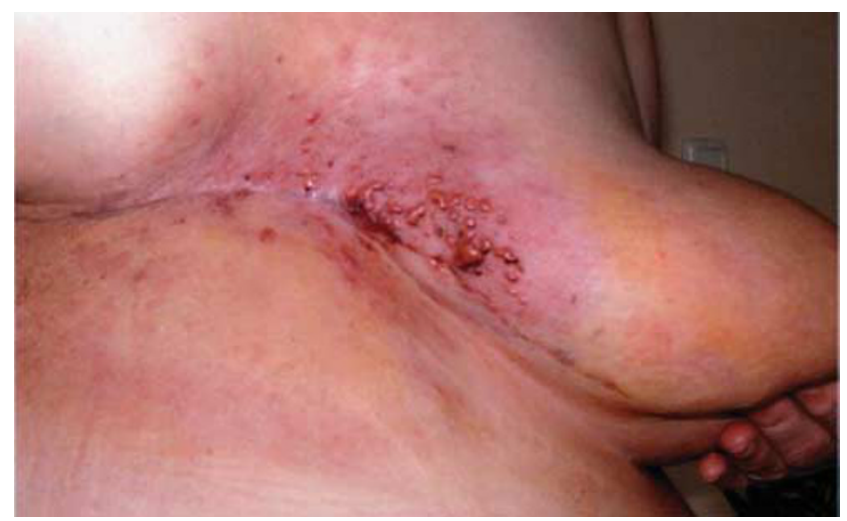

Figure 4 The patient in Case 66 developed an erythematous and indurated plaque with numerous papules and nodules of varying size. hemangioma were noted in Cases 43 and 61 (Figure 6). Intravascular papillary structures lined by atypical endothelial cells were detected in Case 58. A predominantly epithelioid cytomorphology of neoplastic endothelial cells was noted in Cases 62 and 64, whereas spindle-shaped tumor cells predominated in Case 65. The mitotic rate ranged from 2 mitoses in 10 high-power fields in Cases 55 and 59 to 65 mitoses in 10 high-power fields in Case 62, and the Ki-67 index ranged from $10 \%$ in Case 59 to $90 \%$ in Case 64. Ulceration of the overlying epidermis was present in Cases 45, 50, 52, and 60, and areas of confluent tumor necrosis were seen in Cases 52 and 62. Numerous lymphocytes were seen in Cases 51, 53,60 , and 63.

Immunohistochemically, positive nuclear staining of neoplastic endothelial cells for MYC was seen in all but one case (Case 55), and for prox-1 in all but five cases (Cases 44, 46, 58, 62, and 63). Interestingly, the endothelial cells in small vascular structures in the vicinity of the obvious cutaneous angiosarcoma, that were lined by atypical endothelial cells, stained positively for MYC as well (Figure 6). In contrast, cytologically uniform endothelial cells lining small and dilated vascular structures in the periphery showed no expression of MYC. FISH analysis of all cases detected amplification of $M Y C$ in the vast majority of tumor cell nuclei (Table 2). In four cases (Cases 43, 45, 51, and 52), small and dilated vessels in the periphery, lined by cytologically uniform endothelial cells, were examined by FISH analysis as well; however, no MYC amplification was detected in these vessels.

\section{Discussion}

Breast-conserving surgery for the treatment of earlystage breast cancer and in situ lesions is usually combined with adjuvant radiation therapy. It has been shown that radiogenic lymphangiogenesis of the affected skin occurs during the first year after therapy, and that an increased number of podoplanin-positive lymphatic vessels and of CD68-positive histiocytes showing expression of vascular endothelial growth factors can be found. ${ }^{7}$ An important complication of radiotherapy after breast-conserving surgery represents the development of atypical and malignant vascular neoplasms of the skin, and although the exact incidence is unknown, it seems that there has been an increase of postradiation vascular neoplasms. In addition to obvious postradiation cutaneous angiosarcomas, characterized by a significant morbidity and mortality, atypical vascular lesions after radiotherapy for breast cancer have been described under various designations, including atypical vascular proliferation, benign lymphangiomatous papule, acquired (progressive) lymphangioma, acquired lymphangiectasis, and lymphangioma circumscriptum. ${ }^{4}$ Clinically, postradiation atypical vascular lesions tend to occur in 

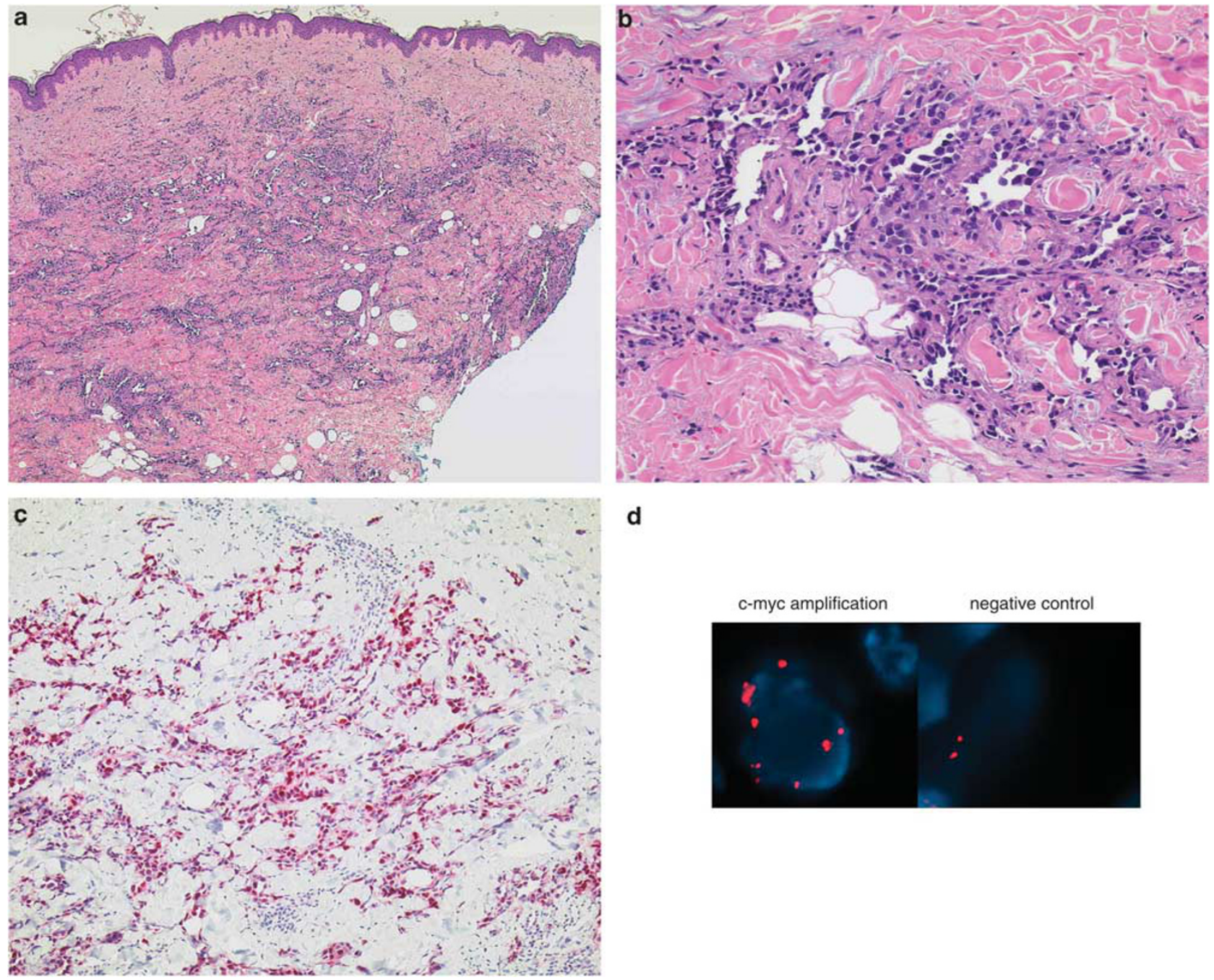

d

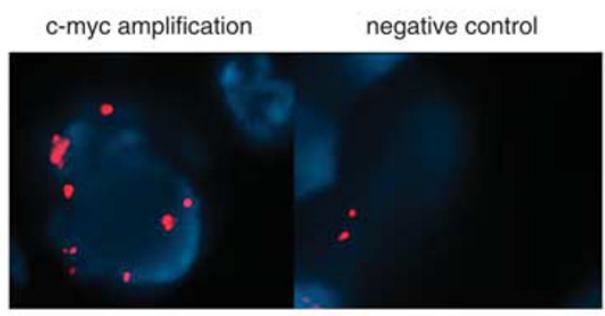

Figure 5 The biopsy of Case 42 shows features of a moderately differentiated cutaneous angiosarcoma. The neoplasm is composed of infiltrating and anastomosing vascular structures lined by atypical endothelial cells (a). Endothelial tumor cells contain enlarged and hyperchromatic nuclei. Note focal endothelial multilayering (b). Immunohistochemically, a strong nuclear staining of endothelial tumor cells for MYC is seen (c), and FISH analysis reveals MYC amplification (d).

slightly younger patients and after a shorter time interval after radiation in comparison to postradiation angiosarcomas and typically present as small solitary papules or nodules. However, in about $20-50 \%$ these lesions present as multiple synchronous or metachronous lesions or even as large plaques, and may recur locally. ${ }^{8}$

Recently, a larger series of 56 radiation-induced vascular lesions arising in 36 patients has been reported. ${ }^{9}$ The vascular neoplasms have been described as relatively well-circumscribed, mainly intradermal lesions with micropapillary tufts and architectural and/or cytologic atypia in 10 cases. Of the patients with follow-up information, five patients developed new vascular lesions in the same area, and of the 10 patients with lesions showing atypical histological features two developed local recurrences. None of the patients showed progression to cutaneous angiosarcoma, and the authors concluded, that postradiation atypical vascular lesions are characterized by a benign clinical course. In contrast, other authors reported that cases of postradiation atypical vascular lesions may show progression to cutaneous angiosarcoma in some cases of subsequent local recurrences, and emphasized that atypical vascular lesions rather represent true precursor lesions. ${ }^{10,11}$ In one of these studies, a broader morphological spectrum of postradiation vascular lesions had been reported, and it had been shown that the risk of tumor progression is associated with the morphological features. Atypical vascular lesions that are composed of small, irregularly dispersed capillary-sized vascular structures, and represent complex vascular neoplasms with involvement of deeper dermal structures, have a higher risk than superficially located, lymphangioma-like lesions composed of dilated, thin-walled lymphatic vessels. ${ }^{11}$ Given the significant clinical, histological, and immunohistochemical overlap between atypical and obvious malignant postradia- 

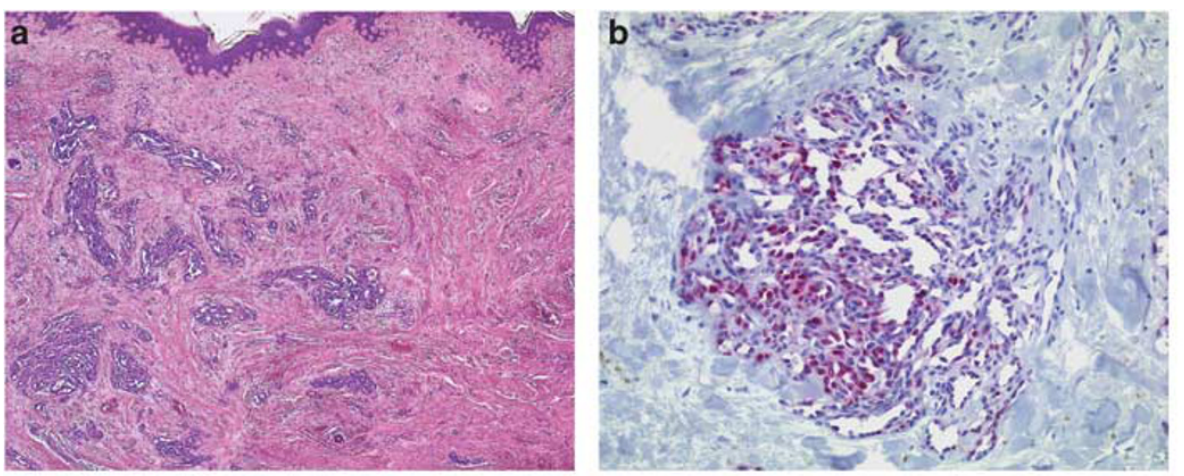

C
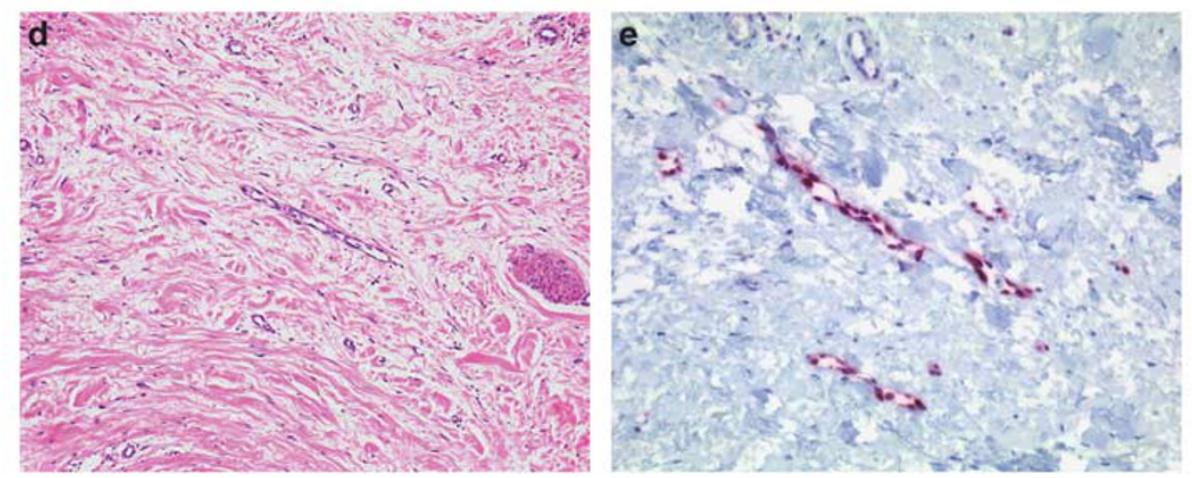

Figure 6 Cellular tufts composed of narrow vascular structures are seen in Case 43 (a). Numerous tumor cells stain positively for MYC (b), and FISH analysis reveals MYC amplification (c). Small vessels in the vicinity of the obvious angiosarcomatous areas were lined by slightly atypical endothelial cells (d), which stained strongly positive for MYC (e).

tion vascular lesions, it seems very problematic to classify reliably individual cases.

The MYC oncogene is a basic helix-loop-helix and leucine zipper transcription factor that is well known for its role in cell proliferation, cellular differentiation, and apoptosis. Importantly, $M Y C$ is also known to stimulate angiogenesis and may promote invasion and metastasis. ${ }^{12}$ MYC deregulation by amplification has been noted in many solid tumors, and recent work emphasizes its role as an important anticancer target. ${ }^{13}$ In regard to soft tissue sarcomas, MYC overexpression and increased $M Y C$ copy numbers are frequent in high-grade chondrosarcomas, ${ }^{14}$ epithelioid sarcomas of the proximal type,${ }^{15}$ in higher-grade myxoid liposarcomas, ${ }^{16}$ and have an adverse prognostic impact in leiomyosarcomas of soft tissues. ${ }^{17}$

A recent study reported $M Y C$ high-level gene amplification in postradiation cutaneous angiosarcomas and in chronic lymphedema-associated cutaneous angiosarcomas (so-called secondary angiosarcomas), whereas so-called primary angiosarcomas of skin, soft tissues, bone, and visceral organs were negative. ${ }^{6}$ Interestingly, high-level amplification of $M Y C$ was found in 55\% of secondary angiosarcomas in this study and was not associated with tumor grade and clinical prognosis. Although primary and secondary angiosarcomas are indistinguishable on morphological ground, they represent genetically different neoplasms. Most recently, a consistent $M Y C$ amplification has been reported in radiation-associated cutaneous angiosarcomas, but not in atypical vascular lesions after radiotherapy for breast cancer. ${ }^{18}$ In this study, the authors reported high-level $M Y C$ amplification at 8 q24 region in $100 \%$ of secondary angiosarcomas, and found a gene amplification of FLT4 in $25 \%$ of cases analyzed. The latter result is of therapeutic interest, and in some patients, in this study showing an amplification of MYC and FLT4, a complete or partial response to sorafenib has been reported.

Our results confirm these previous findings in a large case series. We found amplification of $M Y C$ by FISH analysis in all 25 cases of postradiation cutaneous angiosarcomas, whereas no amplification was detected in atypical vascular lesions following radiotherapy of breast cancer, in so-called primary angiosarcomas of skin and soft tissues, and in control cases. In seven atypical vascular proliferations worrisome morphological features were seen; however, in none of these cases an expression or an amplification of MYC was present, and none of these cases showed a progression to an angiosarcoma. In all, except one case, immunohistochemical stainings for MYC reflected the presence or absence of MYC amplification, emphasizing the use of immunohistochemical stainings in questionable cases. In addition, it has been shown in selected cases that small newly formed vessels in the vicinity of obvious angiosarcomatous areas that were lined by atypical endothelial cells showed an expression for MYC as well. In contrast, no MYC amplification 
has been found by FISH analysis in small newly formed vessels in the periphery of the specimens that were lined by cytologically uniform endothelial cells. These findings suggest that immunohistochemical stainings for MYC are very helpful for mapping of these lesions and for exact control of tumor-free margins. The homeobox gene Prox-1 has an important role in the differentiation of lymphatic vessels ${ }^{19}$ and represents a master control gene that is involved in re-programming of blood vascular to lymphatic endothelial cells. ${ }^{20}$ Considering the increase of lymphatic vessels shortly after radiotherapy, ${ }^{7}$ it is not surprising that the endothelial cells of most cases of analyzed postradiation cutaneous angiosarcomas and of many atypical vascular lesions after radiotherapy stained positively for prox-1, as it has been shown in our study. The lack of prox-1 expression in reactive lesions remains unclear.

Although the presence or absence of $M Y C$ amplification as well as the immunohistochemical expression of MYC clearly separated malignant and atypical postradiation cutaneous vascular neoplasm in our and in previous studies, ${ }^{18}$ and are of considerable diagnostic importance, the pathogenesis of these neoplasms and their relationship are still unclear. Given the lack of $M Y C$ amplification in postradiation atypical vascular lesions, their role as true precursor lesions for postradiation cutaneous angiosarcomas could be questioned, and it could be speculated that atypical vascular lesions have a distinct pathogenesis. ${ }^{18}$ However, it has been shown convincingly that patients with postradiation atypical vascular lesions may develop further lesions, and that these lesions may recur and may even rarely progress to frank cutaneous angiosarcoma. ${ }^{10,11}$ It would be interesting to investigate these rare cases of atypical vascular proliferation showing progression to angiosarcoma in regard to the expression and amplification of MYC, as we assume that $M Y C$ amplification is the molecular event in this progression. For practical purposes, complete excision of postradiation atypical vascular lesions and close follow-up of affected patients are mandatory. Postradiation cutaneous angiosarcomas have to be treated aggressively, and recent knowledge about molecular changes may provide the basis for an additional target therapy of advanced neoplasms.

In summary, we have shown that postradiation cutaneous angiosarcomas are characterized by expression and amplification of $M Y C$, whereas reactive and atypical vascular lesions after radiotherapy do not show these findings. In addition, immunohistochemical stainings for MYC are not only helpful in daily diagnostic work but are also of additional help in mapping of these neoplasms and of careful control of tumor-free margins. Further studies are necessary to clarify the exact relationship of atypical and malignant cutaneous vascular lesions after radiotherapy of breast cancer, to control the predictive value of MYC stainings in preneo- plastic and precursor lesions, and to examine the exact response of advanced neoplasms to targeting therapy. Although the exact incidence is unknown, it seems that the change of therapy of early forms of breast cancer increases the incidence of atypical and malignant cutaneous vascular lesions, and that these therapeutic options have to be discussed critically in some instances.

\section{Acknowledgement}

We appreciate the expert technical assistance of Elke Binot and Ellen Paggen.

\section{Disclosure/conflict of interest}

The authors declare no conflict of interest.

\section{References}

1 Diaz-Cascajo C, Borghi S, Weyers W, et al. Benign lymphangiomatous papules of the skin following radiotherapy: a report of five new cases and review of the literature. Histopathology 1999;35:319-327.

2 Requena L, Kutzner H, Mentzel T. Benign vascular proliferations in irradiated skin. Am J Surg Pathol 2002;26:328-337.

3 Fineberg S, Rosen PP. Cutaneous angiosarcoma and atypical vascular lesions of the skin and breast after radiation therapy for breast cancer. Am J Clin Pathol 1994;102:757-763.

4 Brenn T, Fletcher CDM. Postradiation vascular proliferations: an increasing problem. Histopathology 2006;48:106-114.

5 Santi R, Cetica V, Franchi A, et al. Tumour suppressor gene TP53 mutations in atypical vascular lesions of breast skin following radiotherapy. Histopathology 2011;58:455-466.

6 Manner J, Radlwimmer B, Hohenberger P, et al. MYC high level gene amplification is a distinctive feature of angiosarcomas after irradiation or chronic lymphedema. Am J Pathol 2010;176:34-39.

7 Jackowski S, Janusch M, Fiedler E, et al. Radiogenic lymphangiogenesis in the skin. Am J Pathol 2007;171:338-348.

8 Weaver J, Billings SD. Postradiation cutaneous vascular tumors of the breast. A review. Sem Diagn Pathol 2009;26:141-149.

9 Gengler C, Coindre JM, Leroux A, et al. Vascular proliferations of the skin after radiation therapy for breast cancer: clinicopathologic analysis of a series in favour of a benign process. A study from the French Sarcoma Group. Cancer 2007;109:1584-1598.

10 Brenn T, Fletcher CDM. Radiation-associated cutaneous atypical vascular lesions and angiosarcoma clinicopathologic analysis of 42 cases. Am J Surg Pathol 2005;29:983-996.

11 Patton KT, Deyrup AT, Weiss SW. Atypical vascular lesions after surgery and radiation of the breast. A clinicopathologic study of 32 cases analyzing histologic heterogeneity and association with angiosarcoma. Am J Surg Pathol 2008;32:943-950. 
12 Brooks TA, Hurley LH. The role of supercoiling in transcriptional control of $M Y C$ and its importance in molecular therapeutics. Nat Rev 2009;9:849-861.

13 Soucek L, Whitfield J, Martins CP, et al. Modelling Myc inhibition as a cancer therapy. Nature 2008;455:679-683.

14 Morrison C, Radmacher M, Mohammed N, et al. Myc amplification and polysomy 8 in chondrosarcoma: array comparative genomic hybridization, fluorescent in situ hybridization, and association with outcome. J Clin Oncol 2005;23:9369-9376.

15 Lualdi E, Modena P, Debiec-Rychter M, et al. Molecular cytogenetic characterization of proximal-type epithelioid sarcoma. Genes Chromosomes Cancer 2004;41:283-290.

16 Schneider-Stock R, Boltze C, Jager V, et al. Elevated telomerase activity, c-Myc, and hTERT mRNA expression in association with tumour progression in malignant lipomatous tumours. J Pathol 2003;199: 517-525.

17 Tsiatis AC, Herceg ME, Keedy VL, et al. Prognostic significance of c-Myc expression in soft tissue leiomyosarcoma. Mod Pathol 2009;22:1432-1438.

18 Guo T, Zhang L, Chang NE, et al. Consistent MYC and FLT4 gene amplification in radiation-induced angiosarcoma but not in other radiation-associated atypical vascular lesions. Genes Chromosomes Cancer 2011;50:25-33.

19 Rodriguez-Niedenführ M, Papoutsi M, Christ B, et al. Prox-1 is a marker of ectodermal placodes, endodermal compartments, lymphatic endothelium and lymphangioblasts. Anat Embyol 2002;204:399-406.

20 Hong YK, Harvey N, Noh YH, et al. Prox1 is a master control gene in the programm specifying lymphatic endothelial cell fate. Dev Dyn 2002;225:351-357. 